\title{
Glucocorticoid feedback control of corticotropin in the hypoxic neonatal rat
}

\author{
Hershel Raff and Lauren Jacobson ${ }^{1}$ \\ Endocrine Research Laboratory, Aurora St Luke's Medical Center, Medical College of Wisconsin, Milwaukee, Wisconsin 53215, USA \\ ${ }^{1}$ Center for Neuropharmacology and Neurosciences, Albany Medical College, Albany, New York 12208, USA \\ (Requests for offprints should be addressed to H Raff who is now at Endocrinology, St Luke's Physician's Office Building, 2801 W. KK River Pky, Suite 245, \\ Milwaukee, Wisconsin 53215, USA; Email: hraff@mcw.edu)
}

\begin{abstract}
The objective of this study was to determine the effects of manipulating glucocorticoid negative feedback on acute ACTH and corticosterone responses to corticotropin-releasing hormone $(\mathrm{CRH})$ injection in 7-day-old rats exposed to normoxia or hypoxia from birth. Chemical adrenalectomy was achieved with aminoglutethimide, and glucocorticoids were replaced with a low dose of dexamethasone. Hypoxia per se increased basal plasma corticosterone and attenuated the plasma ACTH response to CRH. Aminoglutethimide per se decreased plasma corticosterone and strongly increased basal plasma ACTH and anterior pituitary POMC gene expression. Dexamethasone partially attenuated elevations in basal plasma
\end{abstract}

ACTH due to aminoglutethimide in both normoxic and hypoxic pups, but inhibited anterior pituitary POMC expression and $\mathrm{CRH}$-induced plasma ACTH only in hypoxic pups. Despite this inhibition, hypoxic pups treated with both dexamethasone and aminoglutethimide still exhibited a significant CRH-induced increment in plasma ACTH, which was lacking in hypoxic pups not treated with either dexamethasone or aminoglutethimide. We conclude that ACTH responses to acute stimuli in hypoxic neonatal rats are prevented by ACTH-independent increases in corticosterone, rather than by intrinsic hypothalamic-pituitary hypoactivity. Journal of Endocrinology (2007) 192, 453-458

\section{Introduction}

Hypoxia is a common neonatal stress leading to significant short-term distress and long-term complications (Frankel \& Stevenson 1987, Friedman \& Fahey 1993, Low et al. 1993, Rubaltelli et al. 1998). Successful adaptation to neonatal hypoxia requires a coordinated physiological response, including an increase in the release of glucocorticoids from the adrenal cortex (Hanukoglu et al. 1995). Understanding the mechanisms by which the resulting increase in glucocorticoid secretion occurs, as well as the physiological impact of this increase in glucocorticoids, will aid in devising strategies to mitigate the short- and long-term effects of neonatal hypoxia. We have previously demonstrated that the neonatal rat exposed to hypoxia from birth has increased plasma corticosterone that is driven by sympathetic input to the adrenal cortex rather than by corticotropin (ACTH; Raff et al. 2003a, 2004). It is possible that this ACTH-independent increase in corticosterone in the neonate exposed to chronic hypoxia from birth is a mechanism to increase circulating glucocorticoids by bypassing the stress-hyporesponsive hypothalamus and/or pituitary.

We have demonstrated that the ACTH response to corticotropin-releasing hormone $(\mathrm{CRH})$ or ether stress was significantly attenuated in the 7-day-old rat exposed to hypoxia from birth (Raff et al. 2003b). We hypothesized that this attenuated pituitary corticotroph response was due to the negative feedback effects of the aforementioned ACTHindependent, sympathetically driven increase in corticosterone. This hypothesis is supported by the evidence that increased sensitivity to glucocorticoid negative feedback is one of the possible mechanisms contributing to the stresshyporesponsive period in the neonatal rat (Walker et al. 1986b, Proulx et al. 2001, Schmidt et al. 2005).

The present study evaluated the hypothesis that the attenuated ACTH response to $\mathrm{CRH}$ in the 7-day-old neonatal rat pup exposed to hypoxia from birth, is due to the ACTH-independent increase in corticosterone. Because it is virtually impossible to adrenalectomize hypoxic neonatal rats with any expectation of survival, we induced a chemical adrenalectomy with aminoglutethimide (Lerner et al. 1984) and then provided different levels of glucocorticoids by means of a vehicle or low-dose dexamethasone injection (Proulx et al. 2001). We have used corticotroph responses to aminoglutethimide and $\mathrm{CRH}$, in the presence or absence of dexamethasone to assess the sensitivity of hypothalamicadrenal-pituitary axis (HPA) axis in normoxic vs hypoxic 7-day-old rat pups to the removal or imposition of glucocorticoid negative feedback. 


\section{Materials and Methods}

The animal protocol was approved by the Institutional Animal Care and Use Committee of Aurora Health Care. Timed pregnant, Sprague-Dawley rats (Harlan, Indianapolis, IN, USA, $n=24)$ at 14 days gestation were obtained and maintained on a standard diet and water available ad libitum (0600-1800 h lights on). Immediately after parturition (days 21-22), dams and their pups were continuously exposed to either normoxia $\left(21 \% \mathrm{O}_{2}\right)$ or hypoxia $\left(12 \% \mathrm{O}_{2}\right)$ in an environmental chamber, as described in detail previously (Thomas \& Marshall 1995, Raff et al. 2000, 2003a). The experimental design is shown in Fig. 1. On postnatal day (PND) 6 at $1600 \mathrm{~h}$, pups were separated into four pretreatment groups, with all pups from a given litter assigned to the same pretreatment. The four pretreatments, each consisting of two i.p. injections with a time gap of $14 \mathrm{~h}$, were as follows: (1) Aminoglutethimide $(400 \mathrm{mg} / \mathrm{kg}$ aminoglutethimide tartrate in $5 \mathrm{ml} / \mathrm{kg}$ saline at $1600 \mathrm{~h}$ of PND 6, followed by $5 \mathrm{ml} / \mathrm{kg}$ saline at $0600 \mathrm{~h}$ of PND 7); (2) Dexamethasone $(5 \mathrm{ml} / \mathrm{kg}$ saline at $1600 \mathrm{~h}$ of PND 6, followed by $5 \mu \mathrm{g} / \mathrm{kg}$ dexamethasone phosphate in $5 \mathrm{ml} / \mathrm{kg}$ saline at $0600 \mathrm{~h}$ of PND 7); (3) Aminoglutethimide plus Dexamethasone (each given at the time and dose indicated above); or (4) Vehicle (5 ml/kg saline at both times). Pups were weighed before each pretreatment injection. Aminoglutethimide and Dexamethasone were obtained from Sigma.

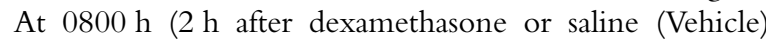
injection), some pups within each litter (Basal) were decapitated and trunk blood was pooled (three pups/sample). Each pool was considered $n=1$ for statistical analysis. Pituitary glands were quickly removed and the anterior lobe was dissected from the neurointermediate lobe. The anterior pituitary lobes of the three pups were pooled for each sample and frozen in liquid nitrogen. Each pool was considered $n=1$ for statistical analysis. The remaining pups $(+\mathrm{CRH})$ within each litter were weighed and injected i.p. with $10 \mu \mathrm{g} / \mathrm{kg}$ of CRH (Bachem/Peninsula Labs, San Carlos, CA, USA diluted in phosphate-buffered saline, $10 \mu \mathrm{l} / \mathrm{g}$ body weight).
The CRH-injected pups were decapitated $30 \mathrm{~min}$ later. Each litter, therefore, provided four pooled plasma samples (two basal; two + CRH) and one to two pooled basal anterior pituitary samples (not every anterior pituitary was successfully retrieved). A vehicle control for $\mathrm{CRH}$ injection was omitted because injection stress does not activate the neonatal HPA axis (Walker et al. 1986a, Arai \& Widmaier 1991).

Plasma ACTH and corticosterone were measured by RIA as described previously (Raff et al. 2003a,b). Pituitary proopiomelanocortin (POMC) mRNA was assessed by northern analysis as described previously (Jacobson et al. 1997, Raff et al. 2003b). ACTH data were log-transformed before analysis of variance to achieve a normal distribution. Data were analyzed by three-factor analysis of variance followed by Duncan's multiple range test.

\section{Results}

Figure 2 shows the ACTH and corticosterone levels achieved before (basal) or $30 \mathrm{~min}$ after $\mathrm{CRH}$ injection $(+\mathrm{CRH})$ in 7-day-old rats exposed to normoxia vs hypoxia from birth and treated with vehicle, aminoglutethimide, and/or dexamethasone. ACTH and corticosterone responded significantly to $\mathrm{CRH}$ injection in normoxic vehicle-treated rat pups (first pair of bars, top and bottom panels of Fig. 2). As we have previously reported (Raff et al. 2003a, 2004), hypoxic vehicle-treated pups had elevated basal levels of corticosterone without an increase in basal plasma ACTH. Unlike normoxic pups, hypoxic pups did not exhibit a significant, CRH-induced increment in either hormone over basal levels (second pair of bars in both panels of Fig. 2). Aminoglutethimide treatment per se significantly reduced corticosterone and increased basal ACTH; levels of both hormones were comparable between normoxic and hypoxic pups. In contrast to normoxic pups, hypoxic pups demonstrated a significant increase in ACTH in response to CRH after aminoglutethimide treatment (third and fourth pairs of bars, Fig. 2). Dexamethasone per se lowered plasma corticosterone to similar levels and blocked the ACTH response

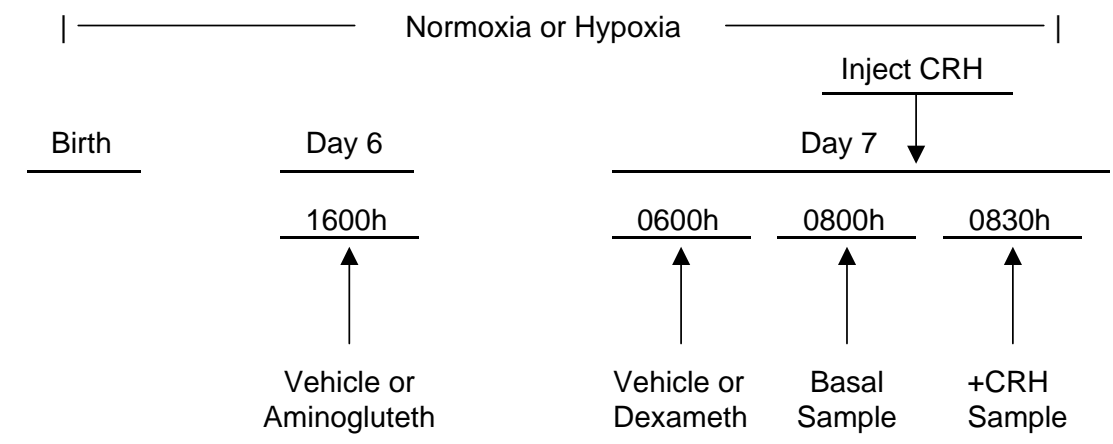

Figure 1 Experimental protocol. At birth, pups were exposed to normoxia or hypoxia for the entire experiment. On day 6 at 0600 h, pups were injected with vehicle or aminoglutethimide. On day 7 at $0600 \mathrm{~h}$, pups were injected with vehicle or dexamethasone. Two hours later, some pups within a litter were sampled (basal). The remaining pups were injected with $\mathrm{CRH}$ and sampled $30 \mathrm{~min}$ later. 


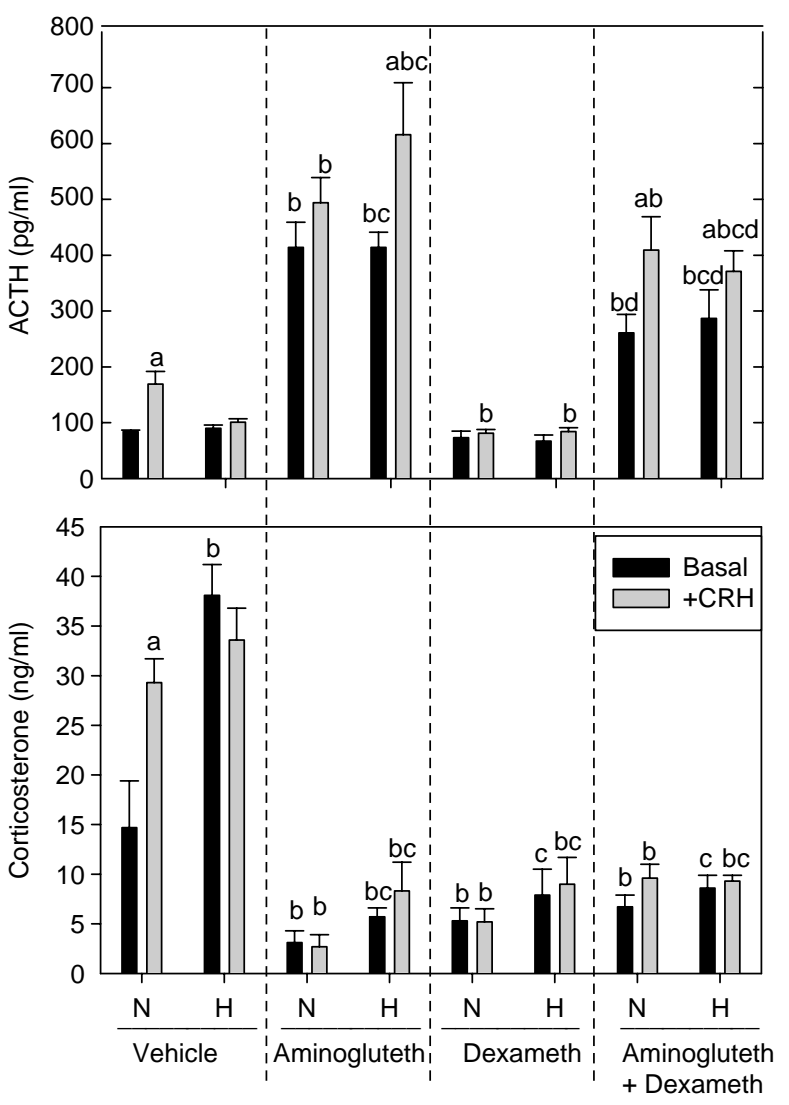

Figure 2 Plasma ACTH (top) and corticosterone (bottom) before (Basal $-0800 \mathrm{~h}$ ) and $30 \mathrm{~min}$ after i.p. injection of $10 \mu \mathrm{g} / \mathrm{kg} \mathrm{CRH} \mathrm{(+}$ $\mathrm{CRH})$ in 7-day-old rat pups exposed to normoxia $(\mathrm{N})$ or hypoxia $(\mathrm{H})$ from birth. Four treatment groups of normoxic and hypoxic pups were studied: Vehicle (two saline injections $(1600 \mathrm{~h}$ the day before and 0600 that day)), Aminoglutethimide injection $(400 \mathrm{mg} / \mathrm{kg}$ at $1600 \mathrm{~h}$ the day before, followed by saline injection at $0600 \mathrm{~h}$ that day), Dexamethasone (saline injection at $1600 \mathrm{~h}$ the day before, followed by Dexamethasone at $0600 \mathrm{~h}$ that day), and aminoglutethimide + dexamethasone (aminoglutethimide injection at $1600 \mathrm{~h}$ the day before, followed by dexamethasone injection at $0600 \mathrm{~h}$ that day). Plasma from three pups was pooled and considered $n=1$.

Each bar is the mean \pm s.E.M. of $n=5-9$ pooled plasma samples. a, Significantly different from basal within the same treatment and the same normoxia or hypoxia group. b, Significantly different from the vehicle normoxia group within the same basal or $\mathrm{CRH}$ group. c, Significantly different from vehicle basal or vehicle CRH respectively within hypoxia. $d$, Significantly different from Aminoglutethimide Basal or Aminoglutethimide+ $\mathrm{CRH}$ respectively within normoxia or hypoxia.

to $\mathrm{CRH}$ in both normoxic and hypoxic pups (fifth and sixth pairs of bars, Fig. 2). Administration of dexamethasone to aminoglutethimide-treated pups resulted in corticosterone levels that were not significantly different from those in pups treated with one drug alone. Aminoglutethimide plus dexamethasone significantly decreased, but did not completely normalize basal plasma ACTH relative to the levels in the corresponding Vehicle controls. CRH induced significant increases over basal levels of ACTH in normoxic and hypoxic pups that had been treated with both dexamethasone and aminoglutethimide (seventh and eight pairs of bars, Fig. 2). The seemingly small difference in plasma ACTH between basal and $+\mathrm{CRH}$ in the hypoxic pups was indeed significant, most likely because the post hoc comparisons factor in rank order as well as differences among mean values. However, CRH-induced ACTH levels in aminoglutethimide-treated pups were significantly reduced by dexamethasone only in hypoxic pups (seventh and eight pairs of bars, Fig. 2).

Figure 3 shows anterior pituitary POMC mRNA levels in the Basal groups of normoxic and hypoxic pups that were given vehicle, aminoglutethimide, dexamethasone, or aminoglutethimide and dexamethasone. Despite differences in basal corticosterone levels (Fig. 2), POMC mRNA was similar between vehicle-treated normoxic and hypoxic pups, and increased to equivalent levels after aminoglutethimide administration (first and second pairs of bars, Fig. 3). Dexamethasone per se did not lower POMC mRNA below the levels in vehicle-treated pups (third pair of bars, Fig. 3). Administration of dexamethasone to aminoglutethimidetreated pups decreased POMC mRNA in hypoxic, but not normoxic pups (fourth pair of bars, Fig. 3).

\section{Discussion}

This study demonstrated in 7-day-old rat pups that (1) aminoglutethimide-induced reductions in corticosterone reveal elevated basal plasma ACTH and ACTH responses to CRH in hypoxic pups and (2) providing glucocorticoid feedback by

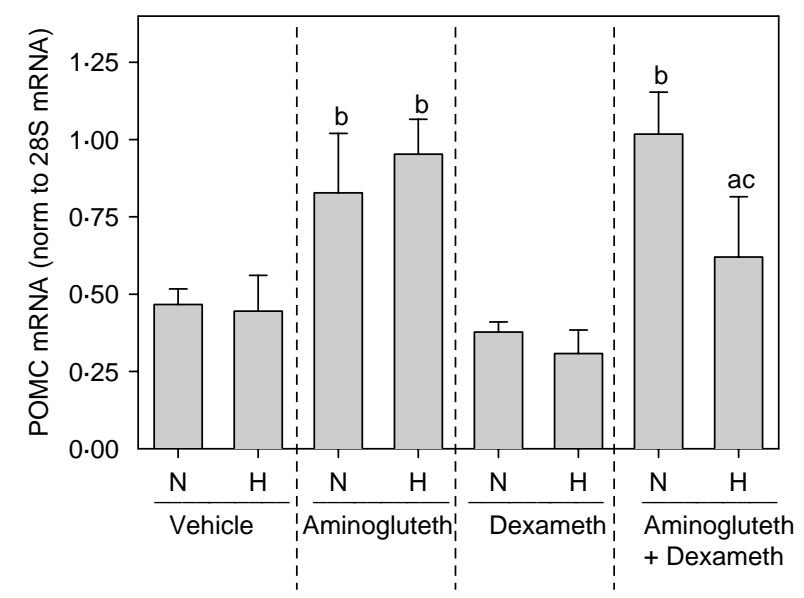

Figure 3 Anterior pituitary POMC mRNA normalized to 28S mRNA in 7-day-old rat pups exposed to normoxia $(\mathrm{N})$ or hypoxia $(\mathrm{H})$ from birth and treated with vehicle, aminoglutethimide, and/or dexamethasone, as described in Fig. 1 and Materials and Methods. Pituitary glands from three pups were pooled and considered $n=1$. Each bar is the mean \pm S.E.M. of four anterior pituitary pooled samples collected from pups in the Basal group. a, Different from normoxic pups within same treatment group. b, Different from the corresponding Vehicle controls within normoxia or hypoxia. c, Different from aminoglutethimide treatment alone within normoxia or hypoxia. 
dexamethasone administration to aminoglutethimide-treated pups resulted in equivalent basal and CRH-stimulated ACTH levels in normoxic vs hypoxic pups, despite differential inhibition of anterior pituitary POMC gene expression.

We have previously demonstrated that hypoxia from birth induced an ACTH-independent increase in corticosterone in 7-day-old rat pups (Raff et al. 2003a). This appeared to be mediated by sympathetic input to the adrenal cortex ( $\mathrm{Raff}$ et al. 2004) and might be enhanced by the development of splanchnic innervation of the medulla at this age (Mikhail \& Mahran 1965, Slotkin \& Seidler 1988). We also previously demonstrated a significantly attenuated ACTH response to $\mathrm{CRH}$ and ether stress in 7-day-old rat pups exposed to hypoxia from birth (Raff et al. 2003b). These differences are not due to differences in corticosteroid-binding globulin or therefore to differences in free corticosterone levels between hypoxic and normoxic pups (Raff et al. 2003a). We confirmed the diminished ACTH response to $\mathrm{CRH}$ in hypoxic pups in the present study. We hypothesized that the ACTHindependent increases in corticosterone suppressed ACTH responses to acute stimuli via negative feedback inhibition. The present study supports that hypothesis.

First, the present study clearly showed that chemical adrenalectomy with aminoglutethimide resulted in large increases in basal ACTH in a manner similar to those observed in older rats (Jacobson et al. 1989). The effects of aminoglutethimide are consistent with the prior evidence that glucocorticoid negative feedback is operational in neonatal rats and may be a component of the etiology of the stresshyporesponsive period (Walker et al. 1986b, Proulx et al. 2001, Schmidt et al. 2005). The functionality of glucocorticoid feedback in neonates was further confirmed by dexamethasone administration per se, which inhibited basal corticosterone and CRH-stimulated ACTH release. Moreover, hypoxic pups responded at least as well as normoxic pups to aminoglutethimide-induced decreases in corticosterone, exhibiting increases in basal plasma ACTH, anterior pituitary $P O M C$ gene expression, and CRH-induced ACTH secretion, which were as great or greater than those in normoxic pups. Since normal corticotroph responses to the removal of glucocorticoid feedback require hypothalamic input (Levin et al. 1988, Walker \& Dallman 1993), these results indicate that the attenuated ACTH responses to the stimuli of $\mathrm{CRH}$ or ether stress that we have previously demonstrated in the hypoxic neonatal rat pups are not due to inherent hypothalamic-pituitary hypoactivity. It is also interesting to note that, despite the prior increases in plasma corticosterone, the plasma ACTH rapidly increased after overnight aminoglutethimide, suggesting a rapid recovery from inhibition by chronically elevated glucocorticoids in hypoxic pups.

A relatively low dose of dexamethasone was chosen (Proulx et al. 2001), so as to reduce but not eliminate aminoglutethimide-induced increases in basal ACTH. When pups were treated with both aminoglutethimide and dexamethasone, $\mathrm{CRH}$ administration resulted in equivalent ACTH levels in normoxic vs hypoxic pups. This result suggests that in the absence of differences in circulating glucocorticoids, hypoxia does not alter the neonatal ACTH response to CRH. Our findings also indicate that hypoxia does not specifically decrease responsiveness of the corticotroph to CRH, which is consistent with previous microanatomical studies showing an increase in the number and size of the corticotroph population after hypoxic exposure (Gosney 1984, Kaur et al. 2002). In fact, with aminoglutethimide alone, the $\mathrm{ACTH}$ response to $\mathrm{CRH}$ was larger in hypoxic when compared with normoxic pups.

We also demonstrated that anterior pituitary POMC mRNA levels are increased by aminoglutethimide in the neonatal rat, and that these increases are comparable between hypoxic and normoxic pups. Interestingly, administration of dexamethasone to aminoglutethimide-treated pups, which decreased basal ACTH significantly to similar levels in both normoxic and hypoxic pups, decreased POMC mRNA only in hypoxic and not in normoxic pups. It may be that decreases in POMC mRNA would have been evident in normoxic pups if we had used sampling times later than $2 \mathrm{~h}$. However, at the time points we used, our data clearly show more rapid inhibition of ACTH and POMC in hypoxic pups after aminoglutethimide and dexamethasone treatment. Consistent with the POMC mRNA data, the ACTH response to CRH in aminoglutethimide-treated pups was also only inhibited by dexamethasone in hypoxic pups. The differential suppression of POMC and CRH-induced ACTH secretion in hypoxic pups is unlikely to be due to differences in circulating levels or clearance of dexamethasone, since basal plasma ACTH showed similar inhibition by dexamethasone in both normoxic and hypoxic pups. The apparently greater sensitivity of POMC and ACTH responses to CRH to dexamethasone in aminoglutethimide-treated hypoxic pups is particularly intriguing, given the lack of inhibition of POMC expression by the elevated corticosterone levels in vehicletreated hypoxic pups. We currently cannot distinguish whether this enhanced sensitivity occurs at the corticotroph, hypothalamus, or higher levels in the HPA axis of the hypoxic neonatal rat.

The use of chemical adrenalectomy does introduce potential comfounds. The primary use of aminoglutethimide in this study was as an inhibitor of P450scc, the first step in the steroidogenic pathway (Chabner et al. 1996). However, in addition to inhibiting adrenal steroidogenesis, aminoglutethimide also decreases gonadal steroidogenesis and inhibits aromatase (Chabner et al. 1996). Despite these confounds, aminoglutethimide has been used for experimental adrenalectomy in previous studies (Lerner et al. 1984, Jacobson et al. 1989). Its advantages are several. First, our model is an exposure of neonatal rat pups to hypoxia from birth. General anesthesia and adrenalectomy of neonatal rats under hypoxic conditions is not a viable experimental model. Secondly, aminoglutethimide allows the maintenance of the integrity of the adrenal medulla (Kent \& Parker 1993), which is important in the neonatal adaptation to hypoxia (Hedner et al. 1980, 
Slotkin \& Seidler 1988). Therefore, the theoretical downsides to the use of aminoglutethimide are outweighed by its advantages in this particular experimental model.

In conclusion, we have demonstrated that the attenuation of the ACTH response to acute stimulation in 7-day-old rat pups exposed to hypoxia from birth is most likely due to glucocorticoid negative feedback. Although total corticosterone levels are low in 7-day-old rats when compared with adults, this is most likely due to low corticosteroid-binding globulin levels (Viau et al. 1996, Raff et al. 2003a). In fact, we propose that free (biologically active) corticosterone is actually normal or even increased, accounting for at least a component of the stress-hyporesponsiveness observed by others (Walker et al. 1986b, Proulx et al. 2001, Schmidt et al. 2005). The present findings demonstrate that even if low, the ACTH-independent increases in glucocorticoid levels in hypoxic neonates are capable of suppressing the ACTH response to acute stimuli such as $\mathrm{CRH}$ administration or ether stress (Raff et al. 2003b). Since glucocorticoid therapy is used to treat pulmonary disease and hypoxia in premature and term neonates (Tzukahara et al. 1999), inhibitory effects of exogenous glucocorticoids, in addition to enhanced feedback due to elevated endogenous glucocorticoid secretion, could impair the ability of the neonatal HPA axis to respond to other stresses in the postnatal period. Because glucocorticoid excess in the perinatal period can also permanently alter the regulation of the HPA axis and glucocorticoid-sensitive endpoints (Raff 2004), elucidating the mechanisms of glucocorticoid feedback in the normal and hypoxic neonate will help avoid adverse long-term sequelae of glucocorticoid therapy.

\section{Acknowledgements}

The authors thank Eric Bruder, Peter Homar, Barbara Jankowski, and Rebecca Rokow-Kittell for their expert technical assistance.

\section{Funding}

This study was supported by NIH DK-54685 to H Raff and by intramural funds from Albany Medical College to L J. There are no conflicts of interest to disclose.

\section{References}

Arai M \& Widmaier EP 1991 Activation of the pituitary-adrenocortical axis in day-old rats by insulin-induced hypoglycemia. Endocrinology 129 1505-1512.

Chabner BA, Allegra CJ, Curt GA \& Calabresi P 1996 Antineoplastic agents. In Goodman and Gilman's The Pharmacological Basic of Therapeutics, 9 , pp 1233-1287. Eds JG Hardman \& LE Limbird. New York: McGraw-Hill.

Frankel L \& Stevenson DK 1987 Metabolic emergencies of the newborn: hypoxemia and hypoglycemia. Comprehensive Therapy 13 14-19.
Friedman AH \& Fahey JT 1993 The transition from fetal to neonatal circulation: normal responses and implications for infants with heart disease. Seminars in Perinatology 17 121-196.

Gosney JR 1984 The effects of hypobaric hypoxia on the corticotroph population of the adenohypophysis of the male rat. Journal of Pathology 142 163-168.

Hanukoglu A, Fried D, Nakash I \& Hanukoglu I 1995 Selective increases in adrenal steroidogenic capacity during acute respiratory disease in infants. European Journal of Endocrinology 133 552-556.

Hedner T, Bergman B \& Holmgren M 1980 Adrenal catecholamines during and following hypoxia in neonatal rats. Medical Biology 58 228-231.

Jacobson L, Akana SF, Cascio CS, Scribner K, Shinsako J \& Dallman MF 1989 The adrenocortical system responds slowly to removal of corticosterone in the absence of concurrent stress. Endocrinology 124 2144-2152.

Jacobson L, Zurakowski D \& Majzoub JA 1997 Protein malnutrition increases plasma ACTH and anterior pituitary pro-opiomelanocortin messenger ribonucleic acid in the rat. Endocrinology 138 1048-1057.

Kaur C, Singh J, Peng CM \& Ling EA 2002 Upregulation of adrenocorticotrophic hormone in the corticotrophs and downregulation of surface receptors and antigens on the macrophages in the adenohypophysis following exposure to high altitude. Neuroscience Letters 318 125-128.

Kent C \& Parker KG 1993 Effects of ACTH and aminoglutethimide on the catecholamine content and chromaffin cell morphology of the adrenal medulla in the neonatal rat. Journal of Anatomy 183 601-607.

Lerner A, Lee PC \& Lebenthal E 1984 Chemical adrenalectomy by aminoglutethimide and the pancreas in suckling rats. American Journal of Physiology. Gastrointestinal and Liver Physiology 247 G346-G351.

Levin N, Shinsako J \& Dallman MF 1988 Corticosterone acts on the brain to inhibit adrenalectomy-induced adrenocorticotropin secretion. Endocrinology 122 694-701

Low JA, Froese AB, Galbraith RS, Smith JT, Sauerbrei EE \& Derrick EJ 1993 The association between preterm newborn hypotension and hypoxemia and outcome during the first year. Acta Paediatrica 82 433-437.

Mikhail Y \& Mahran Z 1965 Innervation of the cortical and medullary portions of the adrenal gland of the rat during postnatal life. Anatomical Record 152 431-437.

Proulx K, Clavel S, Nault G, Richard D \& Walker CD 2001 High neonatal leptin exposure enhances brain GR expression and feedback efficacy on the adrenocortical axis of developing rats. Endocrinology 142 4607-4616.

Raff H 2004 Neonatal dexamethasone therapy: short and long-term consequences. Trends in Endocrinology and Metabolism 15 351-352.

Raff H, Bruder ED, Jankowski BM \& Goodfriend TL 2000 Neonatal hypoxic hyperlipidemia in the rat: effects on aldosterone and corticosterone synthesis in vitro. American Journal of Physiology. Regulatory, Integrative and Comparative Physiology 278 R663-R668.

Raff H, Hong JJ, Oaks MK \& Widmaier EP 2003a Adrenocortical responses to ACTH in the neonatal rat: the effect of hypoxia from birth on corticosterone, StAR, and PBR. American Journal of Physiology. Regulatory, Integrative and Comparative Physiology 284 R78-R85.

Raff H, Jacobson L \& Cullinan WE 2003 blevated corticosterone and inhibition of $\mathrm{ACTH}$ responses to $\mathrm{CRH}$ and ether in the neonatal rat: effect of hypoxia from birth. American Journal of Physiology. Regulatory, Integrative and Comparative Physiology 285 R1224-R1230.

Raff H, Lee JJ, Widmaier EP, Oaks MK \& Engeland WC 2004 Basal and ACTH-stimulated corticosterone in the neonatal rat exposed to hypoxia from birth: modulation by chemical sympathectomy. Endocrinology 145 79-86.

Rubaltelli FF, Bonafe L, Tangucci M, Spagnolo A \& Dani C 1998 Epidemiology of neonatal acute respiratory disorders. Biology of the Neonate $747-15$.

Schmidt M, Levin S, Oitzl MS, van der Mark M, Muller MB, Holsboer F \& de Kloet ER 2005 Glucocorticoid receptor blockade disinhibits pituitaryadrenal activity during the stress hyporesponsive period of the mouse. Endocrinology 146 1458-1464. 
Slotkin TA \& Seidler FJ 1988 Adrenomedullary catecholamine release in the fetus and newborn: secretory mechanisms and their role in stress and survival. Journal of Developmental Physiology 10 1-16.

Thomas T \& Marshall JM 1995 A study on rats of the effects of chronic hypoxia from birth on respiratory and cardiovascular responses evoked by acute hypoxia. Journal of Physiology 487 513-525.

Tzukahara H, Watanabe Y, Yasutomi M, Kovata R, Tamura S, Kimura K, Hiraoka M \& Mayumi M 1999 Early (4-7 days of age) dexamethasone therapy for prevention of chronic lung disease in preterm infants. Biology of the Neonate 76 283-290.

Viau V, Sharma S \& Meaney MJ 1996 Changes in plasma adrenocorticotropin, corticosterone, corticosterone-binding globulin, and hippocampal glucocorticoid receptor occupancy/translocation in rat pups in response to stress Journal of Neuroendocrinology 8 1-8.

Walker CD \& Dallman MF 1993 Neonatal facilitation of stress-induced adrenocorticotropin secretion by prior stress: evidence for increased central drive to the pituitary. Endocrinology 132 1101-1107.
Walker CD, Perrin M, Vale W \& Rivier C $1986 a$ Ontogeny of the stress response in the rat: role of the pituitary and the hypothalamus. Endocrinology 118 1445-1451.

Walker CD, Sapolsky RM, Meaney MJ, Vale WW \& Rivier CL $1986 b$ Increased pituitary sensitivity to glucocorticoid feedback during the stress nonresponsive period in the neonatal rat. Endocrinology 119 1816-1821.

Received in final form 13 November 2006

Accepted 15 November 2006 Made available online as an Accepted Preprint 17 October 2006 